\title{
APLICACIÓN DE UN MÉTODO DE CLASIFICACIÓN SINÓPTICA A LA PENÍNSULA IBÉRICA
}

\author{
Domingo F. Rasilla Álvarez \\ Departamento de Geografía, Urbanismo y Ordenación del Territorio \\ Universidad de Cantabria
}

\section{RESUMEN}

Este trabajo presenta un método de clasificación sinóptica aplicado a la Península Ibérica, combinando técnicas multivariadas de reducción y clasificación de la información y una metodología subjetiva con larga tradición en Climatología Sinóptica. El resultado es un calendario diario de 10 tipos de circulación desde 1946 a 1996. Para verificar su bondad se describen algunas características dinámicas y climáticas de cada uno de los tipos sinópticos.

Palabras clave: Climatología Sinóptica, métodos de clasificación, técnicas estadísticas multivariadas.

\section{ABSTRACT}

This paper introduces a synoptic classification scheme, applied to the Iberian Peninsula. It combines multivariate methods for reduction and classification porpouses, coupled with a manual procedure inspired on a well-known synoptic method. The result is a 51year (1946-1996) daily calendar of synoptic maps classified in 10 patterns. Some climatic and temporal characteristics of such types were checked in order to verify its accurancy.

Key words: Synoptic Climatology, classification methods, multivariate statistical techniques.

\section{RÉSUMÉ}

Cet article présente une méthode hybride de classement synoptique appliquée à la Péninsule Ibérique. Elle combine des techniques multivariées de réduction et de classement de l'information et une méthodologie subjective à longue tradition en Climatologie Synoptique. Le résultat est un calendrier journalier de cartes synoptiques groupées en dix types pour la période 1946-1996. Certaines caractéristiques dynamiques et climatiques de chacun des types synoptiques ainsi établis sont décrites afin de vérifier son exactitude.

Mots-clés: Climatologie synoptique, méthode de classement, techniques statistiques multivariées. 


\section{Introducción}

El objetivo de la Climatología Sinóptica es determinar la influencia de la circulación atmosférica sobre fenómenos naturales y antrópicos. En este sentido, su campo de estudio abarca desde la descripción de los climas regionales hasta el análisis de problemas ambientales como la calidad del aire, los recursos hídricos o el cambio climático (BARRY y PERRY, 1973; YARNAL, 1993). Su piedra angular son las clasificaciones sinópticas, que consisten en una tipificación de los distintos estados de la atmósfera. Los criterios para su elaboración son:

- La similitud en las propiedades termodinámicas de una masa de aire (p.e. temperatura, humedad etc...); de ahí se derivan los «tipos de tiempo», un conjunto relativamente homogéneo de ambientes atmosféricos, producto de la interacción de factores dinámicos y geográficos.

- Unas relaciones comunes entre los componentes en una mapa sinóptico (p.e. localización de los centros de acción). El resultado son los «tipos de circulación».

Los tipos de circulación pueden definirse como las configuraciones que adopta un campo meteorológico (presión a nivel del mar, altura del geopotencial) sobre un ámbito espacial concreto. En función del grado de intervención del investigador en el proceso de clasificación se dividen en manuales o «subjetivas», y automáticas u «objetivas». Entre las primeras destacan las de LAMB (1972) para el Reino Unido, PEDELABORDE (1957) para Francia, y BAUR et al (1944) y HESS y BREZOWSKY (1969) para Alemania, cuya adaptación a la Península Ibérica ha dependido de la formación e influencia recibida por cada investigador (OLCINA, 1994). Pese a su reconocida bondad, comparten una serie de inconvenientes: requieren mucho tiempo y esfuerzo (lo cual, salvo excepciones, ha limitado su aplicación a períodos no superiores a 10 ó 15 años), y la subjetividad aumenta su inconsistencia interna, dificultando su duplicación.

Las clasificaciones automáticas están basadas en la aplicación de algoritmos, como el de «correlación» o «suma de cuadrados» (LUND, 1963; KIRCHOFFER, 1973), procedimientos estadísticos multivariados (GALAMBOSI et al, 1995; PETISCO y MARTIN, 1995; ROMERO et al, 2000) y técnicas de inteligencia artificial (BARDOSSY et al, 1995). A diferencia de las anteriores, proporcionan idénticos resultados siempre que no se cambien los parámetros iniciales y se repita el mismo procedimiento, y aplicadas a las bases de datos en rejilla disponibles en la actualidad, permiten obtener catálogos de gran extensión temporal.

En este trabajo se propone un método para clasificar la circulación atmosférica sobre la Península Ibérica combinando las ventajas de los procedimientos subjetivos y de las técnicas objetivas. Para verificar su validez, también se interpretan las propiedades dinámicas de los tipos obtenidos y su significado climático.

\section{Datos y métodos}

\subsection{Preparación de los datos}

Los valores de presión a nivel del mar provienen de la base de datos $d s 195.5$ (NCEP, EEUU), creada a partir de observaciones de superficie interpoladas a una malla de puntos equidistantes sobre una proyección estereográfica polar. La ventana extraída, 75 nodos entre los paralelos $30^{\circ}$ y $60^{\circ} \mathrm{N}$ y los meridianos $30^{\circ} \mathrm{W}$ y $15^{\circ} \mathrm{E}$, mantiene su espaciado original (aproximadamente $3,75^{\circ}$ ). La matriz inicial se dispuso siguiendo una modalidad $\mathrm{S}$, es decir, cada nodo se convirtió en una variable mientras cada mapa diario se convirtió en 
un caso. Para que los valores extremos no distorsionasen los resultados finales, nodos con valores superiores a \pm 5 desviaciones típicas de su promedio diario fueron eliminados. Estas valores extremos, junto con las lagunas detectadas (menos del 0,3\% de la serie original, en su mayoría con una duración de 1 día), fueron rellenados con el promedio entre el día anterior y el posterior. Para cubrir lagunas iguales o superiores a 2 días se recurrió a las predicciones obtenidas por modelos autorregresivos, aplicados a los 365 días anteriores y posteriores al ausente; la media de ambos modelos sirvió como relleno.

Para resaltar las oscilaciones causadas por la circulación a escala sinóptica y eliminar los ciclos anual y semianual se aplicó un filtro consistente en el cálculo del valor medio de la presión atmosférica en cada uno de los mapas diarios. Posteriormente, se suavizó ese promedio aplicando una media móvil de 13 días, extrayéndose finalmente la diferencia entre cada punto de la malla y el valor suavizado correspondiente a ese día (HEWITSON y CRANE, 1992).

También se han utilizado datos diarios de temperatura máxima y mínima y precipitación total procedentes de observatorios de la red sinóptica del INM y del Servicio Meteorológico de Portugal, obtenidos de la base de datos Global Summary of the Day (NCDC, EEUU).

\subsection{Métodos}

Las técnicas multivariadas comenzaron a aplicarse en Climatología Sinóptica en los años 60 (LORENZ, 1967; KUZBACH, 1967), pero no se popularizaron hasta la década de los 80 , en buena parte por la generalización de los ordenadores personales y la disponibilidad de bases de datos en rejilla. Las técnicas utilizadas con más frecuencia son el Análisis en Componentes Principales y el Análisis de Conglomerados.

La aplicación del Análisis en Componentes Principales persigue varios fines. El primero es la reducción de la información, que en este caso supone identificar y cuantificar las áreas de variabilidad sinóptica más importantes en el ámbito espacial considerado; los procesos de menor escala (subsinópticos y mesoescalares), así como los errores que pudieran subsistir en los datos originales son filtrados. El segundo es la eliminación de la multicolinearidad asociado a campos meteorológicos continuos. Para la extracción de los componentes principales se usa normalmente una matriz de correlación, que impide que las áreas de máxima variabilidad dominen los eigenvectores. Posteriormente, los componentes seleccionados se rotan con un algoritmo VARIMAX para evitar las «configuraciones de Buell» (BUELL, 1979).

La siguiente fase involucra la aplicación de un análisis de conglomerados (cluster analysis), denominación de un conjunto de técnicas multivariadas cuyo propósito es dividir una población en grupos cuya composición y número son desconocidos a priori, sirviéndose de su grado de similitud respecto a unos atributos o características. En el caso que nos ocupa, estos atributos son las amplitudes de los componentes principales, que a su vez cuantifican la magnitud e importancia de esas zonas de variabilidad sinóptica sobre el mapa de presiones de un día concreto.

Los algoritmos utilizados con mayor frecuencia en este tipo de análisis son los jerárquicos (Average linkage, Ward, etc...); entre sus ventajas se encuentra la posibilidad de controlar el proceso según diversos niveles de agrupamiento. Sin embargo, no están exentos de inconvenientes, como su rigidez (una vez asignado un caso a un grupo la clasificación no puede modificarse) o el efecto de «bola de nieve» (génesis de un grupo con un número desproporcionadamente alto de elementos). Aunque estos algoritmos han sido perfeccionados (BUNKERS et al, 1996), su implementación es costosa, limitando su aplicabilidad sobre bases de datos de gran tamaño. 
Como alternativa se suele combinar una fase exploratoria, en la que se obtiene mediante un algoritmo jerárquico una partición preliminar, y otra confirmatoria, usando otro convergente (DAVIS y KALKSTEIN, 1990), entre los que ha alcanzado preeminencia el denominado $k$-means. Éste clasifica las observaciones en un número predefinido de grupos; a medida que se incorporan nuevos casos, su centroide se recalcula tantas veces como sea necesario hasta que no es posible asignar más observaciones, o sea, cuando todos los casos pertenecen al grupo cuyo centroide se encuentra más próximo. Esta naturaleza iterativa optimiza la composición de los grupos y consigue una partición robusta, siempre y cuando las «semillas» (casos iniciales sobre los que se construye la clasificación) se elijan adecuadamente (MILLIGAN y COOPER, 1980).

La elaboración de este tipo de clasificaciones sinópticas no esta exenta de decisiones subjetivas iniciales (tamaño de la ventana, densidad de la rejilla) que condicionan todo el proceso subsiguiente. Pero además existen otros problemas relacionados con el propio objeto de estudio, como es la elección del número de grupos y su significado climático. La atmósfera es dinámica, por lo que la transición de unos estados a otros es habitualmente paulatina; en realidad, las clasificaciones sinópticas constituyen una división artificial con objeto de mejorar nuestro conocimiento de la circulación atmosférica y generalizar una respuesta (HUTH, 1996). En los procedimientos automáticos, el número y naturaleza de los grupos sólo reflejan relaciones matemáticas definidas por alguna medida de similitud (pe. la distancia euclidiana), tomando cada día como un caso independiente, y sin relación a priori con el fenómeno o proceso natural estudiado, es decir, el investigador tiene poco control sobre el proceso de clasificación.

En recientes trabajos la mejora de la capacidad de discriminación de los tipos sinópticos se apoya en clasificaciones previas de campos de variables de superficie (CORTEREAL et al, 1998; PETISCO y MARTÍN, 1996). No obstante, el reparto espacial de las precipitaciones se modifica a medida que el centro de acción se desplaza, pero sin romper la morfología general de la configuración atmosférica. Las últimas investigaciones desarrollan métodos «híbridos» (FRAKES y YARNAL, 1997) en los que el número y la composición de los grupos es establecido inicialmente por el investigador sobre una muestra reducida, a partir de su conocimiento de la dinámica atmosférica y su repercusión sobre el tiempo en una región; las técnicas automáticas clasifican el resto de la población.

Cuadro 1

\section{DENOMINACIÓN DE LOS TIPOS SINÓPTICOS}

\begin{tabular}{|l|l|l|}
\hline Tipo 1 & ACE & Anticiclón Cantábrico-Europa Occidental \\
\hline Tipo 2 & ZON & Circulación zonal. \\
\hline Tipo 3 & AZO & Anticiclón de las Azores. \\
\hline Tipo 4 & GRN & «Greenland Above». \\
\hline Tipo 5 & DAT & Dorsal Atlántica. \\
\hline Tipo 6 & CLP & Ciclónico Península. \\
\hline Tipo 7 & BLA & Bloqueo Atlántico. \\
\hline Tipo 8 & RTC & Retrógrada Continental. \\
\hline Tipo 9 & AIM & Anticiclón Ibero-Mediterráneo. \\
\hline Tipo 10 & ACT & Anticiclón Centrado. \\
\hline
\end{tabular}


ACE

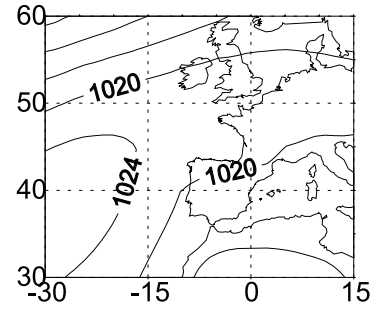

GRN

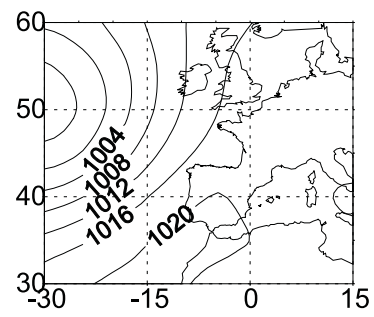

BLA

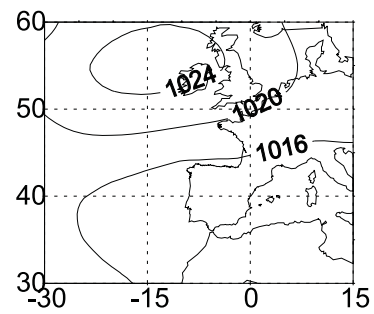

ZON

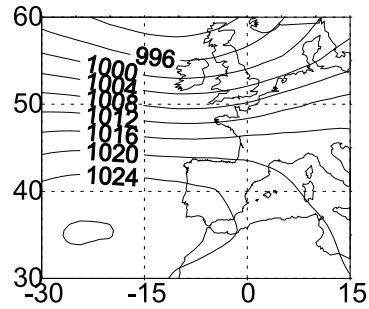

DAT

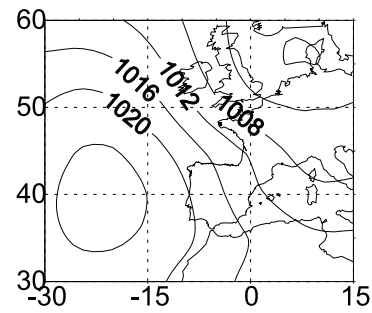

RTC

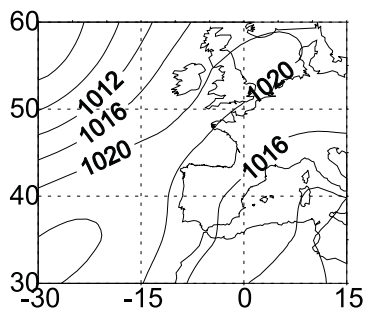

ACT

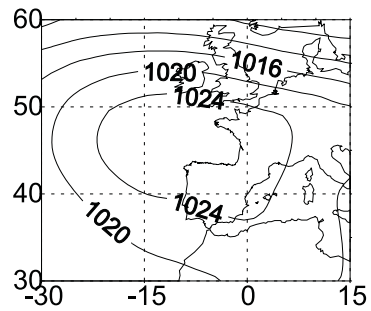

$\mathrm{AZO}$

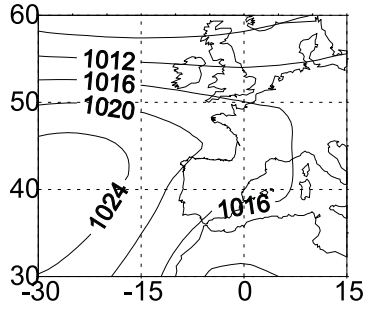

CLP

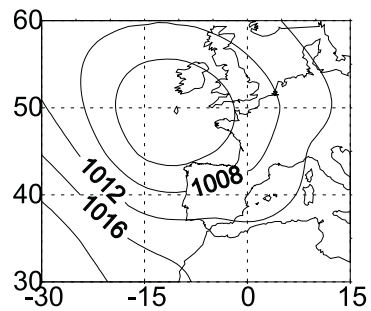

AIM

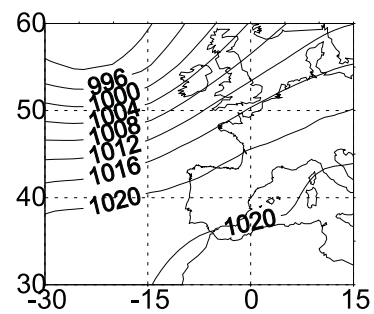

Figura 1. Configuración promedio (hPa) de uno de los tipos de circulación. 
El método propuesto en este trabajo adopta esta última orientación metodológica. Comporta una primera clasificación subjetiva de la circulación atmosférica sobre la Península Ibérica durante el período 1977-1991, inspirada fundamentalmente en criterios cinemáticos: las categorías se seleccionaron atendiendo al movimiento y trayectoria («steering») de los centros de acción, la curvatura del campo de presión superficial y la procedencia de la advección (FERNÁNDEZ GARCÍA, 1985). Criterios adicionales fueron las condiciones meteorológicas de superficie y la consulta de estudios de naturaleza similar (MICHELANGELI et al, 1995; ZHANG et al, 1997). El número de grupos fue elegido como compromiso entre la capacidad explicativa de la diversidad climática de la Península Ibérica y su manejabilidad, a partir de otros estudios emprendidos por el autor (cuadro 1 y fig. 1); su denominación proviene de la localización de los centros de acción protagonistas de cada configuración.

De esta fase inicial se obtuvo una clasificación provisional, cuya validez estadística se ratificó mediante un Análisis Discriminante (CANADA, 1989; GALAN, 1989), y que generó las «semillas» que sirvieron para elaborar los centroides de cada uno de los grupos. Éstos constituyeron la base para la clasificación final, aplicando un Análisis de Conglomerados aglomerativo (K-means) al periodo 1946-1996. La estabilidad de la clasificación fue comprobada repitiendo este último proceso cada década por separado, así como fragmentando la base de datos en muestras aleatorias (validación cruzada).

\section{Resultados}

\subsection{Identificación de los componentes principales}

En este tipo de análisis, los componentes principales no equivalen a situaciones sinópticas reales, sino a áreas en las que la presión varía simultáneamente, por lo que es posible interpretarlos en términos de los centros de acción que contribuyen a cada componente y su evolución anual (fig. 2). Un total de 6 componentes, reflejando un $92 \%$ de la varianza original, superaron los test de significación habituales (CATTELL, 1966; OVERLAND y PREISENDORFER, 1982; NORTH et al, 1982):

- El modo dominante de la circulación atmosférica a escala sinóptica sobre la Península Ibérica es atribuible a los anticiclones iberomediterráneos y las depresiones sobre el Mediterráneo Occidental. Su valor es positivo en el semestre invernal y negativo durante la primavera y comienzo del verano.

- El segundo componente reproduce la posición media del Anticiclón de las Azores en invierno, reemplazado en verano por bajas presiones de naturaleza térmica, provenientes del $\mathrm{N}$ de África.

- El tercero hace referencia a las depresiones que atraviesan el N. de Europa o a los anticiclones continentales, siendo las primeras más frecuentes en invierno y los segundos en primavera.

- El cuarto refleja la intensidad del campo de presiones sobre la Península Ibérica. Sus valores mínimos ocurren desde febrero a mayo y los más elevados durante el invierno.

- El quinto refleja las oscilaciones de la presión sobre el Atlántico N, probablemente como reflejo de la evolución de la Depresión de Islandia, y es opuesto al anterior.

- El sexto es la contribución de los anticiclones polares atlánticos y de las depresiones formadas en torno a las Azores, cuyo ritmo anual está marcado por el predominio de condiciones ciclónicas en invierno y anticiclónicas en verano. 


\section{$1^{\circ} \mathrm{CP}$}
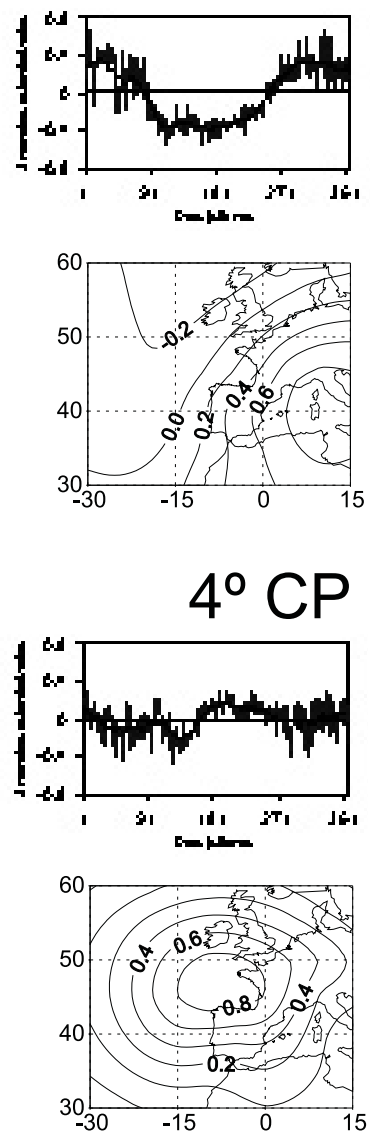
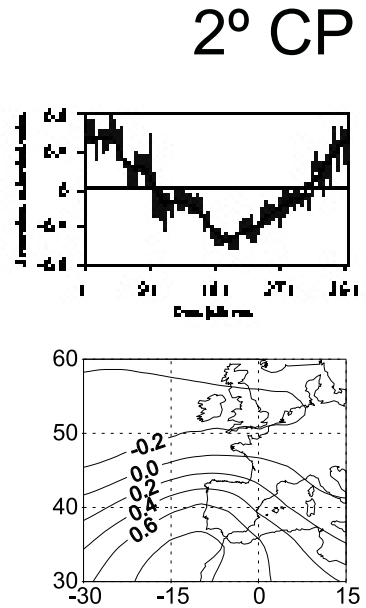

$5^{\circ} \mathrm{CP}$
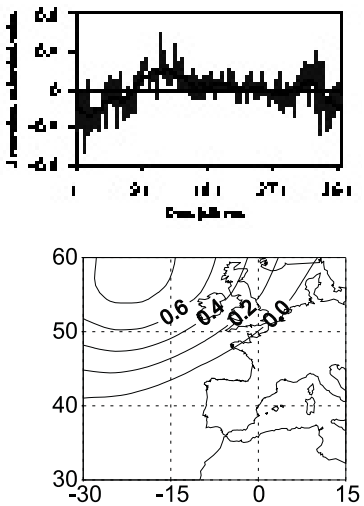

$3^{\circ} \mathrm{CP}$
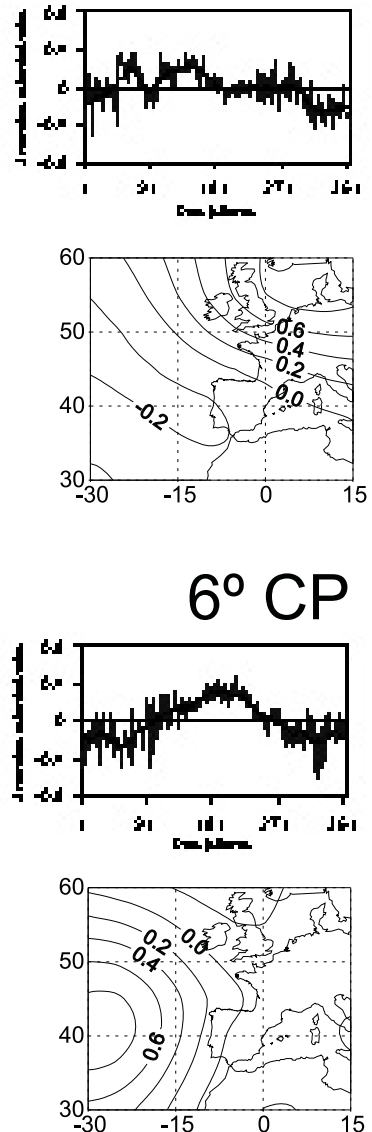

Figura 2. Localización espacial de los componentes principales y evolución estacional.

\subsection{Características dinámicas de los tipos sinópticos}

El grado de estabilidad de la atmósfera se deduce de la presión a nivel del mar (P) y la vorticidad (Z). Situaciones totalmente estables son la 2, la 9 y la 10, mientras la 6 y la 7 pueden definirse como muy inestables. En otras categorías, ambos parámetros se disocian debido una posición excéntrica de algunos centros de acción: el tipo 4 genera un campo de presión moderadamente perturbado, pero una baja vorticidad, al contrario que los tipos 1 y el 3. Estas configuraciones reproducen también diferentes gradientes de presión, y como consecuencia, valores contrastados en la velocidad media del viento geostrófico $(\mathrm{F})$, en función de la ubicación de la Península Ibérica respecto a los centros de acción: los tipos 2 , 5 y 6 están caracterizados por altas velocidades, en contraste con las situaciones de bloqueo (tipos 1 y 7) o con las predominantemente anticiclónicas (9 y 10). 


\section{Cuadro 2}

CARACTERÍSTICAS DINÁMICAS DE LOS TIPOS SINÓPTICOS

\begin{tabular}{|l|c|c|c|}
\hline & PRESIÓN & F & Z \\
\hline Tipo 1 & 1019,7 & 7,64 & 2,61 \\
\hline Tipo 2 & 1022,7 & 10,19 & $-18,36$ \\
\hline Tipo 3 & 1017,9 & 7,84 & 0,20 \\
\hline Tipo 4 & 1018,8 & 9,17 & $-17,67$ \\
\hline Tipo 5 & 1016,8 & 14,22 & $-6,26$ \\
\hline Tipo 6 & 1009,0 & 12,97 & 11,75 \\
\hline Tipo 7 & 1014,6 & 7,58 & 7,31 \\
\hline Tipo 8 & 1017,5 & 9,70 & $-0,69$ \\
\hline Tipo 9 & 1020,0 & 7,01 & $-13,77$ \\
\hline Tipo 10 & 1024,8 & 8,00 & $-13,83$ \\
\hline PROMEDIO & 1018,0 & 9,16 & $-3,56$ \\
\hline$X^{2}$ & 6667,5 & 2675,5 & 6543,0 \\
\hline Sig, & 0,00 & 0,00 & 0,00 \\
\hline
\end{tabular}

Los tipos sinópticos también discriminan claramente la procedencia de las advecciones:

- Los pertenecientes a una circulación de «índice alto» están caracterizados un predominio de las advecciones del $3^{\text {er }}$ (tipos 4,6 y 9) y $4^{\circ}$ cuadrantes (tipos 2 y 5).

- Las circulaciones retrógradas determinan el predominio de flujos del $1^{\text {er }}$ cuadrante, bien consecuencia de células anticiclónicas continentales (tipo 8) o marítimas (tipo $3)$.

- Una circulación de bloqueo desencadena flujos del $2^{\circ}$ cuadrante, procedentes de un bloqueo difluente (cut off high, tipo 7), una gota fría (cut off low, tipo 1), o simplemente por la retirada de la circulación del W a latitudes septentrionales (tipo 10).

Cuadro 3

FRECUENCIA (\%) DE LA DIRECCIÓN DEL VIENTO GEOSTRÓFICO EN SUPERFICIE EN CADA TIPO SINÓPTICO

\begin{tabular}{|r|r|r|r|r|r|r|r|r|r|r|}
\hline & T1 & T2 & T3 & T4 & T5 & T6 & T7 & T8 & T9 & T10 \\
\hline $1^{\text {er }} \mathrm{C}$ & 48,4 & 12,6 & 65,9 & 13,9 & 32,8 & 0,4 & 36,6 & 63,2 & 20,7 & 35,1 \\
\hline $2^{\circ} \mathrm{C}$ & 32,2 & 3,3 & 18,0 & 17,7 & 0,1 & 2,5 & 33,7 & 8,4 & 25,0 & 51,8 \\
\hline $3^{\circ} \mathrm{C}$ & 5,0 & 24,3 & 2,0 & 39,3 & 1,0 & 56,4 & 13,0 & 1,6 & 29,7 & 8,4 \\
\hline $4^{\circ} \mathrm{C}$ & 14,3 & 59,7 & 14,1 & 29,2 & 66,0 & 40,8 & 16,7 & 26,8 & 24,5 & 4,6 \\
\hline
\end{tabular}




\subsection{Variaciones temporales}

La diversidad en el número medio de días pertenecientes a cada tipo sinóptico es un primer rasgo significativo en este catálogo, oscilando entre un máximo de 60 días al año en el caso del tipo $3(17 \%)$ al mínimo protagonizado por el tipo $2(7,2 \%)$. Su variabilidad interanual también es significativa; en el caso del tipo 3 el máximo de 89 días se alcanzó en 1954 frente a un mínimo de 21 casos en 1968. Dicha frecuencia experimenta también variaciones a lo largo del año (fig. 3). Las situaciones inestables muestran su predilección por las estaciones intermedias, mientras las estables dominan en verano; el invierno se configura como una estación relativamente equilibrada, aunque sesgada hacia algunas situaciones estables. Entre éstas últimas pueden distinguirse variantes invernales (tipo 8 y 10), resultado de la expansión del anticiclón de las Azores hacia la Península Ibérica y el Mediterráneo Occidental, y estivales (tipos 1 y 3), en este caso hacia el centro de la cuenca atlántica. Entre las perturbadas, el otoño es más favorable a las configuraciones que producen flujos del SW, mientras que en primavera dominan las depresiones centradas o sobre el Mediterráneo Occidental.

La posición de la Península Ibérica en el borde meridional de la Corriente en Chorro favorece el predominio de circulaciones estables. Esta situación se acentúa cuando el Jet Stream se desplaza hacia el N, como ocurre en verano, aunque en superficie factores geográficos, como el recalentamiento del sustrato peninsular y del Norte de África enmascaran, a través de la creación de una zona de bajas presiones, esa estabilidad. Dicha afirmación parece entrar en contradicción con la conformación de un máximo relativo de situaciones estables durante el invierno, momento en el que los Westerlies alcanzan su posición más meridional. Sin embargo, la Península es afectada por una dorsal en altura; el flujo del oeste gira hacia el Mediterráneo desde el momento en que toma contacto con las costas europeas, a causa de su rozamiento contra el continente, torsión que es facilitada por la tendencia de las masas frías continentales a desplazarse hacia el Mediterráneo aprovechando distintos pasillos orográficos (p.e. el valle del Ródano). Esta torsión favorece la
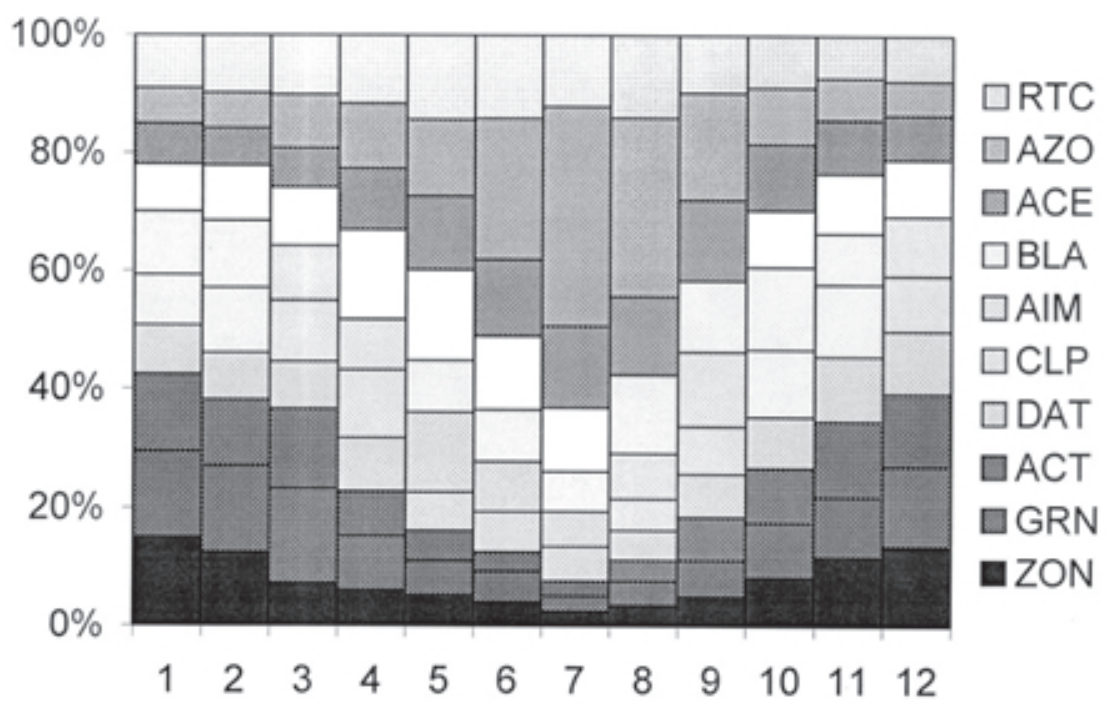

FIGURA 3. Frecuencia media mensual (\%) de los tipos de circulación (1946-1996). 
formación sobre el Mediterráneo Occidental de un centro de bajas presiones (la depresión de Liguria o del Golfo de Génova) y la aproximación del Anticiclón de Azores a la Península Ibérica, cuyo origen dinámico es reforzado por factores térmicos (estabilización de las capas bajas por enfriamiento sobre un sustrato continental). Las situaciones inestables son el resultado de su expansión a través de ondulaciones (vaguadas) o vórtices aislados (gotas), consecuencia de su debilitamiento y ralentización, que tiene mayor auge en primavera, a causa de un debilitamiento de los contrastes térmicos entre las superficies oceánicas y continentales.

Los contrastes en la frecuencia de los tipos dependen estrechamente de su persistencia, que a su vez se vincula a las características de la circulación regional en la que se inserta (cuadro 5). La configuración más persistente es la 3, que reproduce la posición media del Anticiclón de las Azores en verano, mientras en otoño y primavera esta condición recae en circulaciones «lentas», bien bloqueos sobre la Península Ibérica (tipo 10) o Europa Occidental e Islas Británicas (tipo 7), bien configuraciones celulares (tipo 1). Las situaciones más efímeras están insertas en una circulación «rápida» (tipos 2, 4, 5 y 6), aunque su encadenamiento en invierno ocasiona los típicos temporales atlánticos. La menor permanencia de las situaciones inestables es, de nuevo, una consecuencia de su ubicación meridional respecto al Jet Stream, que favorece la llegada de estos centros de acción negativos a partir de la configuración de ondulaciones o vórtices desprendidos del cuerpo principal.

Cuadro 4

PERSISTENCIA (DÍAS CONSECUTIVOS) DE CADA SITUACIÓN SINÓPTICA

\begin{tabular}{|l|r|r|r|r|r|r|r|r|r|r|}
\hline DíAS & T1 & T2 & T3 & T4 & T5 & T6 & T7 & T8 & T9 & T10 \\
\hline 1 & 582 & 325 & 526 & 361 & 434 & 435 & 287 & 612 & 567 & 290 \\
\hline 2 & 354 & 157 & 266 & 191 & 191 & 202 & 151 & 293 & 277 & 153 \\
\hline 3 & 166 & 79 & 137 & 85 & 94 & 96 & 99 & 141 & 99 & 73 \\
\hline 4 & 73 & 37 & 105 & 30 & 27 & 39 & 58 & 74 & 51 & 32 \\
\hline 5 & 21 & 28 & 48 & 31 & 18 & 30 & 36 & 33 & 18 & 24 \\
\hline 6 & 11 & 15 & 45 & 16 & 5 & 14 & 30 & 14 & 9 & 24 \\
\hline 7 & 1 & 2 & 16 & 11 & 4 & 8 & 18 & 4 & 5 & 10 \\
\hline 8 & & 6 & 17 & 5 & 1 & 10 & 13 & 4 & 2 & 4 \\
\hline 9 & & & 10 & 3 & 1 & 3 & 5 & 1 & & 2 \\
\hline 10 & & 2 & 5 & 2 & & & 7 & & & \\
\hline 11 & & & 2 & 2 & & 1 & 4 & 1 & & 3 \\
\hline 12 & & & 1 & 1 & & & & & & \\
\hline 13 & & & 5 & & & & & & & \\
\hline 14 & & & 2 & & & 1 & & & & \\
\hline 15 & & & 1 & & & & & & & \\
\hline+1 & & & 8 & 1 & & & & & & \\
\hline
\end{tabular}


La existencia de secuencias fijas en la transición entre unos tipos y otros define el concepto de regímenes de circulación. Sin embargo, a escala diaria el carácter caótico de la propia circulación atmosférica, y en especial, el comportamiento inestable de sus grandes ondulaciones planetarias, impide los encadenamientos rígidos, de tal manera que el concepto de secuencias o sucesión debe tomarse como una tendencia estadística general, con un elevado número de grados de libertad. La irrupción de tipos sinópticos poco frecuentes, así como el retorno a la misma configuración sinóptica de días previos, proporcionan una secuencia evolutiva discontinua, sometida a cambios bruscos, y por supuesto, de difícil previsión (VAUTARD, 1990; PLAUT y VAUTARD, 1994).

No obstante, no todas las sucesiones están reguladas por el azar. En este sentido, los rasgos esenciales de la evolución de la circulación atmosférica en el SW de Europa vuelven a adquirir una gran relevancia. La pertenencia de la Península Ibérica, al menos durante gran parte del año, al dominio de los Westerlies facilita las transiciones en sentido horario, de ahí que las más frecuentes conforman una secuencia clásica. La circulación zonal próxima a la Península Ibérica es bastante efímera (tipo 2), pasándose rápidamente a otra más lenta y ondulada, en el que la componente predominante será la meridional si en el Atlántico aparece una vaguada y el anticiclón de las Azores se desplaza hacia el E (tipos 9, 4 y 6), o bien septentrional si los mecanismos de ciclogénesis orográfica desvían los Westerlies hacia el Mediterráneo Occidental, mientras sobre el Atlántico la descarga de aire polar se acompañada de una dorsal en altura, que permite el ascenso en latitud de las altas presiones atlánticas y su engarce con las masas frías continentales centroeuropeas.

Otro rasgo que cabe la pena destacar es la alta frecuencia con la que las transiciones configuran un giro del flujo en el mismo sentido de las agujas del reloj. Este hecho es típico de situaciones en las que la circulación es zonal u ondulada (tipos 2, 4, 5 y 6), y tiene que ver con el modo de propagación de las grandes ondas de la circulación general. Bajo condiciones de circulación retrógrada (tipos 1 y 3) o claramente de bloqueo (tipos 7 y 10), las secuencias son más complejas, y en relación con la trayectoria típica de unas depresiones que se dirigen hacia el Mediterráneo desde el Golfo de Cádiz y Mar de Alborán.

Cuadro 5

TRANSICIONES ENTRE TIPOS SINÓPTICOS (EN HORIZONTAL DÍA SIGUIENTE D+1)

\begin{tabular}{|c|r|r|r|r|r|r|r|r|r|r|}
\hline & T1 & T2 & T3 & T4 & T5 & T6 & T7 & T8 & T9 & T10 \\
\hline T1 & & 50 & 303 & 81 & 69 & 108 & 117 & 277 & 125 & 78 \\
\hline T2 & 73 & & 42 & 93 & 49 & 76 & & 53 & 224 & 41 \\
\hline T3 & 359 & 37 & & & 165 & 76 & 90 & 200 & 211 & 56 \\
\hline T4 & 47 & 96 & 3 & & 59 & 131 & 74 & 116 & 109 & 103 \\
\hline T5 & 30 & 161 & 185 & 6 & & 137 & 75 & 42 & 49 & 90 \\
\hline T6 & 105 & 98 & 54 & 185 & 47 & & 97 & 121 & 117 & 15 \\
\hline T7 & 142 & 1 & 90 & 70 & 81 & 88 & & 117 & 12 & 107 \\
\hline T8 & 284 & 35 & 234 & 76 & 174 & 152 & 128 & & 66 & 28 \\
\hline T9 & 89 & 121 & 193 & 167 & 67 & 63 & 13 & 218 & & 97 \\
\hline T10 & 79 & 52 & 90 & 61 & 64 & 8 & 113 & 33 & 115 & \\
\hline
\end{tabular}




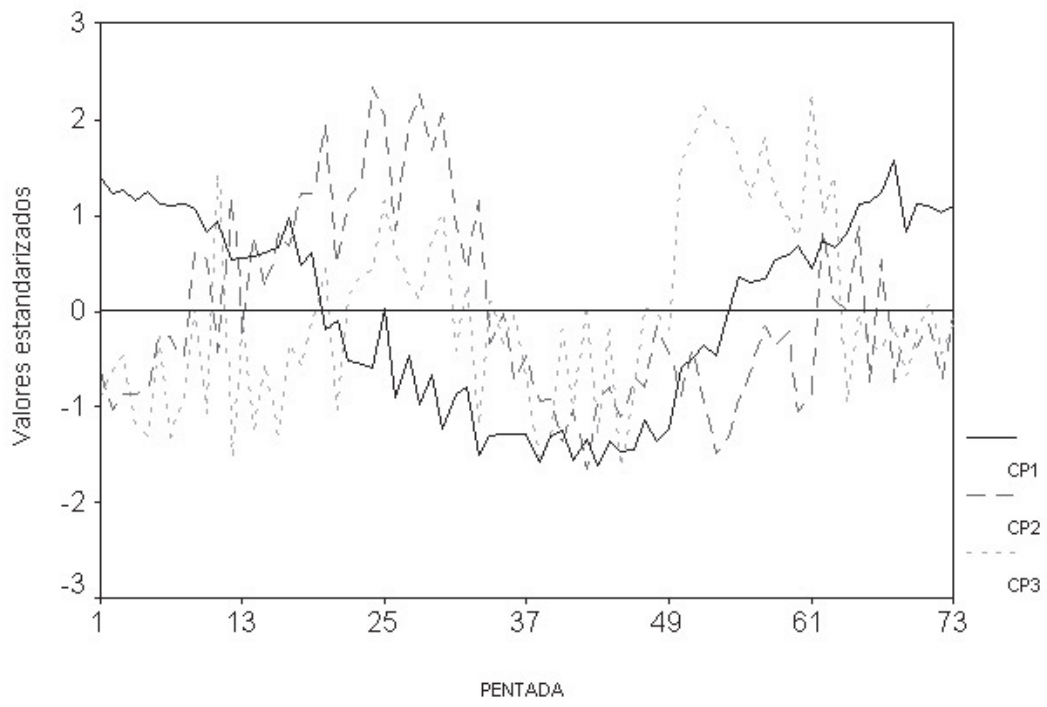

Figura 4. Amplitud de los 3 componentes principales del ciclo anual (en péntadas) de la circulación atmosférica sobre la Península Ibérica.

A partir de la repetida aparición de una serie de configuraciones sinópticas en momentos muy concretos del año, se pueden definir unas «estaciones naturales» $\mathrm{y}$ «singularidades». Para identificar estos periodos se aplicó un análisis en componentes principales a la frecuencia absoluta de los 10 tipos de circulación durante un hipotético año divido en 73 péntadas (MAHERAS, 1988). 3 componentes (cuadro 3 y fig. 4) explican el

\section{Cuadro 6}

PONDERACIONES DE LOS 3 PRIMEROS COMPONENTES PRINCIPALES DE LA FRECUENCIA DE TIPOS SINÓPTICOS A LO LARGO DEL AÑO (PÉNTADAS)

\begin{tabular}{|l|r|r|r|}
\hline & PC1 & PC2 & PC3 \\
\hline Tipo 1 & -0.84 & -0.16 & 0.36 \\
\hline Tipo 2 & 0.91 & -0.13 & -0.20 \\
\hline Tipo 3 & -0.90 & -0.32 & -0.13 \\
\hline Tipo 4 & 0.87 & 0.09 & -0.20 \\
\hline Tipo 5 & 0.66 & 0.26 & 0.10 \\
\hline Tipo 6 & 0.49 & 0.70 & 0.29 \\
\hline Tipo 7 & -0.61 & 0.54 & 0.31 \\
\hline Tipo 8 & -0.73 & 0.36 & -0.36 \\
\hline Tipo 9 & 0.56 & -0.46 & 0.58 \\
\hline Tipo 10 & 0.91 & -0.05 & -0.08 \\
\hline \% var VAR & 58 & 13 & 8 \\
\hline
\end{tabular}


$79 \%$ de la variabilidad; el primero, que constituye el proceso fundamental de la circulación atmosférica sobre la Península Ibérica (58\% de la varianza), opone un régimen invernal (ponderaciones positivas de $\operatorname{los}$ tipos 2, 4, 5, 9 y 10) a otro estival (ponderaciones negativas de los tipos 1, 3, 7 y 8); las transiciones entre ambos ocurren al final de las péntadas 59 (22 de octubre) y 25 (30 de marzo). El segundo (13\%) incide particularmente en aquellas configuraciones con máximos primaverales (tipos 6 y 7), mostrando valores positivos entre las péntadas 33 y 45 (13 marzo a 23 de junio) y 62 y 68 (17 de septiembre a 30 de octubre), y dos negativos, el estival (1 de julio al 16 de septiembre) más intenso que el invernal (1 noviembre a 12 de marzo). Por su parte, el tercer componente (únicamente el $8 \%$ de la varianza) distingue situaciones propicias a flujos de componente meridional (tipos 6, 7, y 9).

En consecuencia, un año medio podría dividirse en las siguientes «estaciones naturales»:

- Un período invernal, entre las péntadas 64 y 18 (12 de noviembre al 31 de marzo), caracterizado por amplitudes positivas del $1^{\text {er }}$ componente y negativas del $2^{\circ}$ y $3^{\circ}$. La llegada del invierno es clara, pero su final constituye un periodo de transición, entre las péntadas 12 y 18, en el que ninguno de los dos componentes se impone con claridad.

- La primavera se ubica entre las péntadas 19 y 35 (desde el 1 de abril al 24 de junio), y en ella las amplitudes positivas del $2^{\circ}$ componente, y un cierto repunte del tercero, son su rasgo distintivo.

- El verano (péntadas 36 a 48 -25 junio a 28 agosto-) corresponde al período de predominio de amplitudes negativas de los 3 componentes.

- A partir de la péntada 49 se inicia el otoño, en el que el $3^{\text {er }}$ componente adquiere valores positivos, acompañado de una recuperación progresiva del $1^{\circ}$.

En comparación con el calendario civil, el invierno se prolonga hasta finales de marzo, mientras el verano se reduce a los meses de julio y agosto. Otro aspecto a destacar es el contraste entre la paulatina evolución de las amplitudes del primer componente, es decir, de la intensidad de los Westerlies, frente a la de los componentes $2^{\circ}$ y $3^{\circ}$, sobre todo durante las estaciones equinocciales, reflejando bruscos reajustes de la circulación hemisférica producidos por las alteraciones en el balance energético planetario.

\subsection{Características climáticas}

Esta clasificación puede validarse también analizando los campos espaciales de precipitación y temperatura vinculados a cada tipo sinóptico, para deducir los posibles mecanismos dinámicos responsables.

Las precipitaciones más abundantes e intensas en la Cornisa Cantábrica aparecen con circulaciones del NW y N (tipos 5 y 8, fig. 5). Las advecciones de poniente (configuraciones 4 y 6) son responsables de las jornadas más lluviosas en la vertiente atlántica, aunque en este conjunto se observa un gradiente latitudinal vinculado a la sustitución de los procesos advectivos asociados a circulaciones zonales, dominantes en las regiones septentrionales (tipo 2) por mecanismos convectivos (tipo 7). En la vertiente mediterránea, la baja frecuencia de días de precipitación y su reducida intensidad no impide eventos excepcionales, sobre todo con las situaciones 1,7 y 8 , cuya características común es la proximidad de un centro de acción cerrado sobre la Península Ibérica o en sus proximidades, y un flujo procedente del E. En todos los casos, la interacción entre relieve y mecanismos dinámicos es clara, mostrando una intensificación a barlovento de las principales cadenas montañosas, y una reducción en zonas bajo abrigo orográfico, particularmente en 

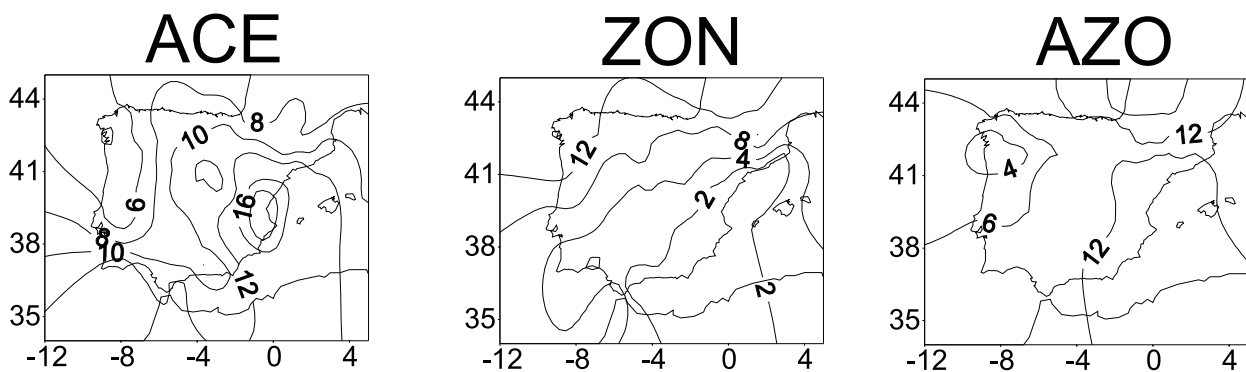

GRN

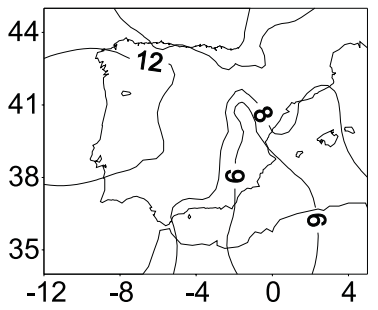

BLA

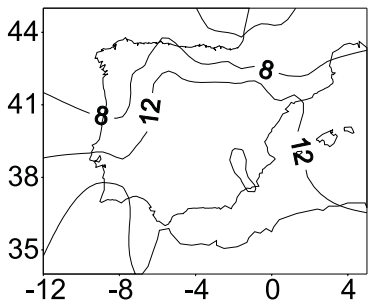

DAT

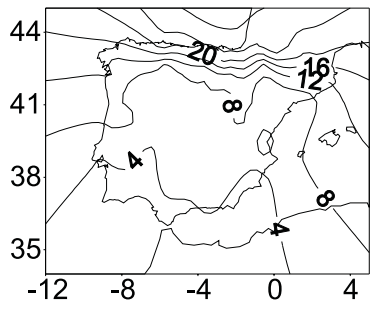

RTC

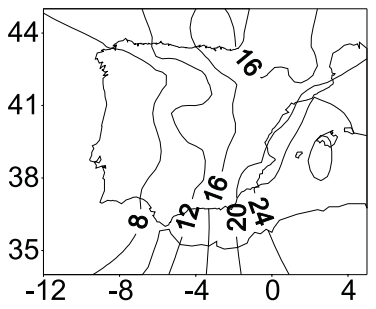

ACT

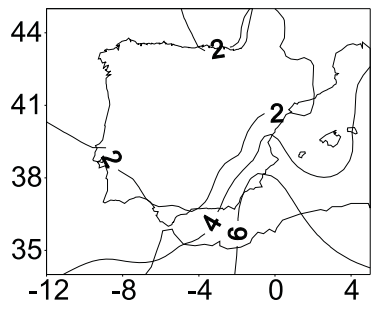

CLP

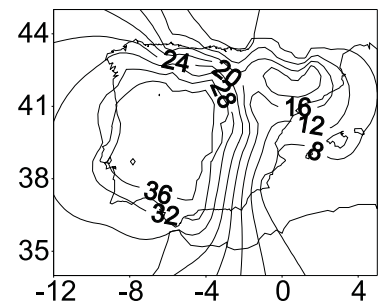

AIM

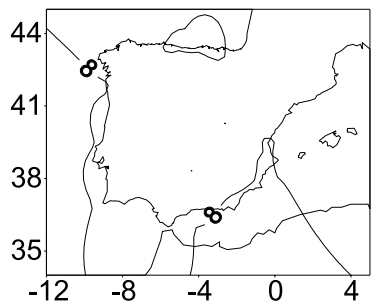

FIgURA 5. Intensidad media de la precipitación diaria (décimas de mm) en cada uno de los tipos sinópticos. 

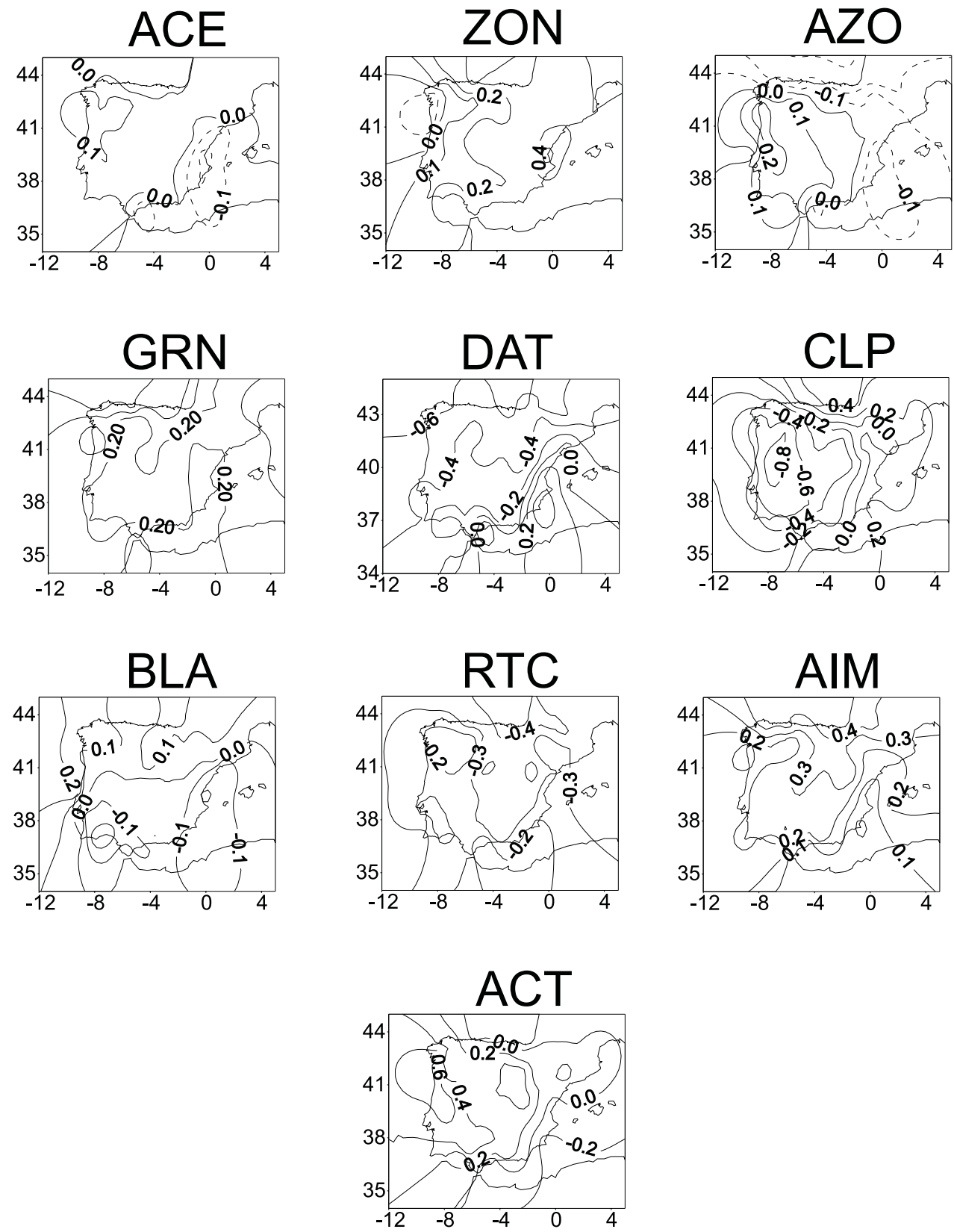

Figura 6. Anomalía media de la temperatura máxima diaria (diferencia en ${ }^{\circ} \mathrm{C}$ respecto a un periodo de 13 días) en cada uno de los tipos sinópticos. 

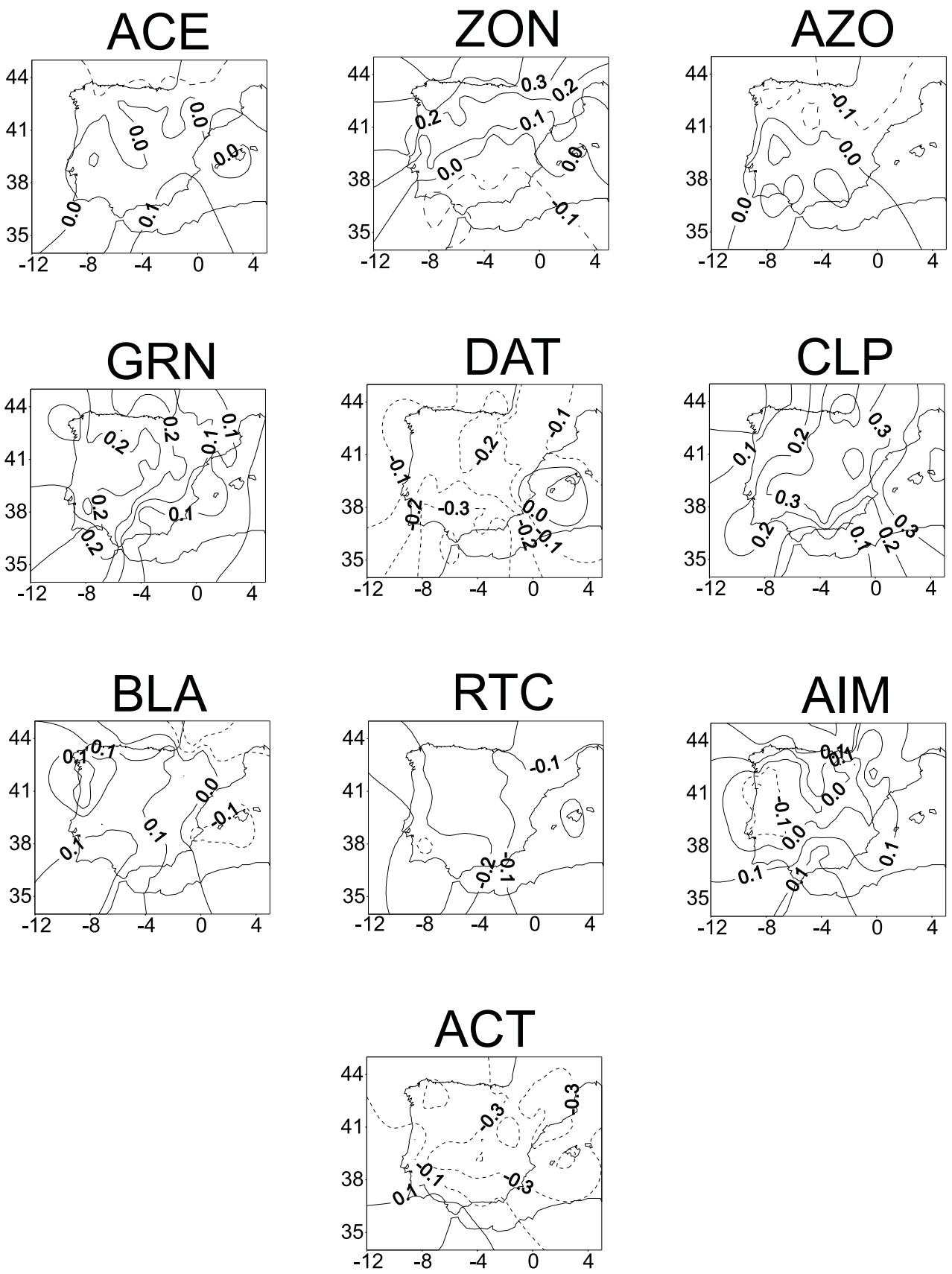

FIgURA 7. Anomalía media de la temperatura mínima diaria (diferencia en ${ }^{\circ} \mathrm{C}$ respecto a un periodo de 13 días) en cada uno de los tipos sinópticos. 
las configuraciones direccionales. En las configuraciones con predominio de la componente convectiva, los movimientos verticales conducen a una homogeneización espacial.

La procedencia de las advecciones controla estrechamente la distribución espacial de las temperaturas, aunque su análisis choca con más dificultades, por la estacionalidad de los tipos sinópticos y la diferente variabilidad diaria de las temperaturas, tanto desde el punto de vista temporal (máxima en invierno, mínima en verano) como espacial (continentalidad versus oceanidad). Al igual que se hizo con la presión, los valores diarios fueron filtrados y estandarizados sobre un intervalo temporal de 13 días.

El componente dinámico es determinante en el comportamiento de las temperaturas máximas (figs. 6 y 7), pero otros factores, de naturaleza geográfica, tienden a enmascarar el comportamiento de las temperaturas mínimas. Los tipos cálidos generalizados están asociados a advecciones meridionales (tipo 10), mientras los fríos aparecen vinculados a tipos septentrionales (tipos 5 y 8). El relieve interviene aportando ciertos matices regionales; por ejemplo, los tipos zonales son templados o moderadamente frescos en la mitad occidental de la Península Ibérica (procedencia marítima de la masa de aire y abundante nubosidad), características que se desvirtúan en el Mediterráneo y en el Cantábrico por «efecto föhn»: es el caso de la situación 5 en Levante (flujo del NW con «Ponent») o de las 4 y 6 en el Cantábrico (flujo del SW con «Sur»). La proximidad a superficies oceánicas con corrientes frías próximas introduce peculiaridades aún más locales, vinculados a la presencia de nieblas estivales (p.e. en la costa occidental de Portugal - Cabo Carvoeiro-o en el Occidente de Asturias —aeropuerto de Ranón-).

\section{Conclusiones}

Este trabajo propone un método para la clasificación de mapas sinópticos diarios, que solventa algunos de los inconvenientes de los métodos automáticos, manteniendo al mismo tiempo las ventajas de los manuales. La metodología contempla la elección subjetiva del número y composición de los grupos a partir de una muestra reducida, a la que se aplica un Análisis Discriminante, clasificándose posteriormente toda la población a través de un Análisis de Conglomerados.

Una serie de criterios confirman la bondad del catálogo propuesto. El primero es la reproducción de configuraciones físicamente distintas, comparables a las sugeridas por otros autores, y fácilmente interpretables en términos de localización de los centros de acción. El segundo es la reproducción de estados atmosféricos en los que se aprecia una clara interacción entre la dinámica atmosférica y las condiciones geográficas. Un último criterio es su capacidad para reproducir la estacionalidad de la circulación atmosférica sobre la Península Ibérica. Dada la concentración de ciertas configuraciones en momentos concretos del año, ha sido posible ajustar la definición tradicional de las estaciones a la evolución real de la circulación atmosférica.

\section{Bibliografía}

CORTE-REAL, J.; QIAN, B. y XU, H. (1998). «Regional Climate Change in Portugal: Precipitation Variability associated with Large-Scale Atmospheric Circulation». Int. Jor. Climatol, 18 (6), pp 619-636.

BARDOSSY, A.; DUCKSTEIN, L. y BOGARDI, Y. (1995). «Fuzzy rule-based classification of atmospheric circulation patterns». Int. J. Climatol, 15, pp. 1087-1097.

BARRY, R.G y PERRY, A.H. Synoptic climatology. Methods and applications. London, Methuen 1973, 273 pp. 
BAUR, F.; HESS, P. y NAGEL, H. Kalendar der GroB wetterlagen Europas 1881-1939. Bad Homburg, Homburg, 1944.

BUELL, C.E. (1979). «On the physical interpretation of empirical orthogonal functions». 6th Conf. on Probability and Statistics in Atmosheric Sciencies, Banff, Alberta, Amer. Meteor. Soc., pp. 112-117.

BUNKERS, M.; MILLER, J. y DeGAETANO, A. (1996). «Delimitation of climate regions in the Northern Plains using an objetive cluster modification technique». J. Climate., 9, pp. 130-146.

CAÑADA TORRECILLA, R. El clima de Extremadura. Tesis Doctoral, Dep. de Geografía, Universidad Autónoma de Madrid, Madrid 1989.

CATELL, R.B. (1966). «The scree test for the number of factors». Multivar. Behav. Res., I, pp. 245276.

DAVIS, R. y KALKSTEIN, L. (1990). «Development of an automated spatial synoptic climatological classification». Int. J. Climatol., 10, pp. 769-794.

FERNÁNDEZ GARCÍA, F. El clima de la Meseta Meridional: los tipos de tiempo. Edit. Universidad Autónoma de Madrid, Madrid 1985.

FRAKES, B. y YARNAL, B. (1997). «A procedure for blending manual and correlation-based synoptic classifications». Int. J. Climatol., 17, pp. 1381-1396.

GALAMBOSI, A.; DUCKSTEIN, L. y BOGARDI, I (1996). «Evaluation and analysis of daily atmospheric circulation patterns of the $500 \mathrm{hPa}$ pressure field over the southwestern USA». Atms. Res., 40, pp. 49-76.

GALAN GALLEGO, E.: Tipos de tiempo anticiclónicos invernales en la España Peninsular y Baleares. Ensayo metodológico. Tesis Doctoral, 3 vol, Madrid 1989, 1405 pp.

HESS, P. y BREZOWSKY, H. Katalog der Grosswetterlagen Europas. Beritche des Deuchen Wetterdienstes Nr. 113, Bd. 15, 2 neu bearbeitete und ergänzte Aufl., Offenbach a. Main, Selbstverlag des Deutschen Wetterdientes, 1969.

HEWITSON, B. y CRANE, R.G. (1992). «Regional climates in the GISS Global Circulation Model and Synoptic-scale Circulation. J. Climate, 5, pp. 1002-1011.

HUTH, R. (1996). «An intercomparison of computer-assisted circulation classification methods». Int. J. Climatol., 16, pp. 893-992.

KIRCHOFFER, W. (1973). «Classification of $500 \mathrm{mb}$ patterns». Arbeitsbericht der Schwizerischen Zentralandstalt., 45, $16 \mathrm{pp}$.

KUZBACH, J.E. (1967). «Empirical eigenvectos of sea level pressure, surface temperature and precipitation complexes over North America». J. Appl. Meteorol., 6, pp. 792-802.

LAMB, H.H. (1972). «British Isles weather types and a register daily sequence of circulation patterns, 1861-1971». Geophysical Memoir, 110, Met. Office, London, 85 pp.

LORENZ, E. N. (1967). Emprical orthogonal functions and statistical weather prediction. Scientific Report 1, Statistical Forecasting Project, Department of Meteorology, Massachussets Institute of Technology, 48 pp.

LUND, L. (1963). «Map pattern classification by statistical methods». J. Appl. Meteorology, 2, pp. 56-65.

MAHERAS, P. (1988). «Delimitation of the summer-dry period in Greece according to the frequency of weather types». Theor. Appl. Climatol., 39, pp. 171-176.

MICHELANGELI, P.A.; VAUTARD, R. y LEGRAS, B. (1995). «Weather regimes: recurrence, and quasi-stationarity». J. Atm. Scienc, 52, pp. 1237-1256.

MILLIGAN, G.W. y COOPER, M.C. (1985). «An examination of the effect of six types of error perturbation on fifteen clustering algorithms». Psychometrica, 45, pp. 325-342.

NORTH, G.R.; BELL, T.L. y COHALAN, R.F. (1982). «Sampling errors in estimation of empirical orthogonal functions». Mon. Wea. Rev., 110, pp. 699-706.

OLCINA CANTOS, J. (1994). «Métodos de clasificacion sinóptica en España. Revisión y propuesta». Estudios Geográficos, 215, pp. 357-387.

OVERLAND, J.E. y PREISENDORFER, R.W. (1982). «A significance test for principal components applied to cyclone climatology». Mon. Wea. Rev, pp. 110, 1-8.

PEDELABORDE, P. Le climat du Bassin Parisien. De. Th. Génin, París 1957. 
PETISCO DE LARA, E. y MARTIN HERREROS, J.M. Caracterización de la circulación atmosférica en la Península y Baleares». Servicio de Análisis e Investigación del Clima, informe nº5, INM, Madrid 1995.

PLAUT, G. y VAUTARD, R. (1994). «Spells of low-frequency oscillations and weather regimes in the Northern Hemisphere». J. Atm. Scienc, 51, pp. 210-235.

ROMERO, R.; SUMNER, G.; RAMIS, C. y GENOVÉS, A. (1999). «A classification of the Atmospheric Circulation Patterns Producing Significant Daily Rainfall in the Spanish Mediterranean Area». Int. J. Climatol., 19, pp. 765-785.

VAUTARD, R. (1990). «Multiple weather regimes over the North Atlantic: analysis of precursors and successors». Mon. Wea. Rev, 118, pp. 2056-2081.

YARNAL, B. Synoptic climatology in Environmental Analysis. Studies in Climatology Series. Belhaven Press, London, 1993, 195 pp.

ZHANG, X.; WANG, X. y CORTE-REAL, J. (1997). «On the relationship between daily circulation patterns and precipitation in Portugal». J. Geophys. Res., 102, pp. 13495-13507. 
